\title{
Control of urinary schistosomiasis on Zanzibar (Unguja Island): a pilot evaluation of the educational impact of the Juma na Kichocho health booklet within primary schools
}

\author{
JR Stothard/+', P Mook*, AF Mgeni**, IS Khamis**, AN Khamis**, D Rollinson
}

Department of Zoology, Natural History Museum, Cromwell Road, London SW7 5BD, UK *Department of Infectious and Tropical Diseases, The London School of Hygiene and Tropical Medicine, London, UK **Helminth Control Laboratory Unguja, Helminth Control Programme, Ministry of Health and Social Welfare, Zanzibar

To improve health education within primary schools, the health education booklet Juma na kichocho was evaluated during a study within 5 schools using key-informant questionnaires that recorded children's knowledge and attitude (KA) towards schistosomiasis before and after daily structured-use of booklets. A total of 229 schoolchildren (114 boys : 115 girls) of between 11 and 15 years of age were interviewed and re-assessed after a working school week. Existing and putative booklet-induced changes in KA scores for schistosomiasis were compared directly against equivalent KA scores for malaria. In total $47.4 \%$ of children were already aware that schistosomiasis was a water-borne disease while only 10.5\% knew of its exact aetiology; after booklet intervention these levels increased to 54.6 and $15.7 \%$, respectively. The majority of children still failed, however, to realise that re-infection could take place soon after treatment. While a positive increase was observed for children's total KA questionnaire scores for both malaria and schistosomiasis after booklet intervention, these were not statistically significant. In the context of control, further educational efforts are needed to promote and guide behavioural change, especially in relation to reduction of environmental water contact.

Key words: health education - knowledge and attitude - information education and communication - malaria Schistosoma haematobium

Schistosomiasis continues to be a scourge of the health of people living in sub-Saharan Africa with a yearly ascribed death rate of over 200,000 (van der Werf et al. 2003). The global burden of the disease is presently under revision (King et al. 2005) and there has been renewed support for control. Several recent initiatives are focused upon provision of chemotherapy with praziquantel, often in conjunction with medications for other diseases (Molyneux et al. 2005), to ensure the prevention and containment of schistosomiasis-induced morbidity (Fenwick 2006). Administration of medications, with focus usually upon treatment of school-aged children, is primarily facilitated through use of school-based resources (Southgate et al. 2005).

Urinary schistosomiasis, caused by infection with Schistosoma haematobium, is a major public health problem in Zanzibar and is locally known as kichocho (Stothard et al. 2002a). In contrast to the widespread distribution of the disease on Pemba Island (Albonico et al. 1997, Lwambo et al. 1997), the distribution of urinary schistosomiasis on Unguja is clearly restricted within the distribution of the only suitable local intermediate snail host, Bulinus globosus (Stothard \& Rollinson 1997). Prevalence of the disease by school is strongly correlated with proximity to $B$.

Financial support: The Health Foundation, UK ${ }^{+}$Corresponding author: r.stothard@nhm.ac.uk.

Received 25 May 2006

Accepted 26 June 2006 globosus habitats and with levels of children's local water contact as ascertained by interrogative questionnaires (Stothard et al. 2002a, b). Earlier questionnaire methods revealed that awareness of kichocho was initially low allowing much room for improvement (Stothard et al. 2002b).

In October 2003, the President of Zanzibar launched the Kick out Kichocho control programme on Unguja. The programme receives support through a partnership of donors: The Health Foundation, The African Development Bank and the Schistosomiasis Control Initiative and is implemented by the Helminth Control Laboratory Unguja (HCLU) and monitored by the Natural History Museum. The HCLU also acts as a referral dispensary for diagnosis and treatment of those requiring or seeking treatment. Operating within the known endemic zone for schistosomiasis, the aims of Kick out Kichocho are to carry out school-based treatment with praziquantel, to provide health education and coordination of community sensitisation and to investigate longer-term possibilities for transmission control by manipulation of snail populations.

To improve health education within primary schools, the health education booklet Juma na Kichocho is to be utilised as a novel teaching aide. The booklet's primary role is to provide health education messages pertinent for kichocho in a child-friendly format to encourage ease of learning. The comic booklet can be downloaded electronically from http://www.who.int/wormcontrol/ education_materials/tanzania/en/swahili_comic_ juma.pdf. In its original hardcopy inception produced by CHEPE (St Fons, France), it is a 13 page durable booklet in comic strip layout relating the personal story of Juma, a 
young boy who learns about kichocho from his teachers and local health centre staff. Specific messages in the booklet include the recognition of the disease's primary signs and symptoms e.g. passing of blood in urine and pain upon micturition, the provision of information concerning the life-cycle and transmission of S. haematobium e.g. a disease of both man and aquatic snails and is caught by playing in contaminated freshwater ponds and streams, as well as taking appropriate control measures e.g. compliance with treatment and promotion of behavioural change to reduce water contact and transmission of disease. In a visually appealing and non-didactic manner, the booklet hopes to educate the child by enforcing key health messages set within an appropriate cultural content and story setting. Within the African context, the Juma na Kichocho booklet has also been used as templates for other medical strip cartoons used by Unicef which include: malaria, mosquito-nets, safe injections and yellow-fever.

Delivery of health education is known to be an important aspect within schistosomiasis control programmes (Engels \& Mpitabakana 1989, Kloos 1995, Aryeetey et al. 1999 , Schall \& Diniz 2001) and health propaganda is today better known as Information, Education and Communication (IEC) materials. Measuring the tangible impact of health education can be problematic (Lansdown et al. 2002) but, when successful, health education can play a major role in initiating and guiding behavioural change (Teesdale 1986, Yuan et al. 2000).

This pilot study aimed to assess the immediate impact of the Juma na Kichocho booklet upon the knowledge and attitude of school children in order to devise optimal strategies for more wide scale implementation and evaluation.

\section{MATERIALS AND METHODS}

Study area - A total of 5 primary schools were selected to be representative of rural and semi-urban environments on Unguja where originary schistosomiasis in schoolaged children was either at low $(\sim 10 \%)$ or at moderate-to-high $(\geq 25 \%)$ levels of endemicity. The schools, with associated parasitological prevalence of S. haematobium $(S . h$.) and Global Positioning System (GPS) co-ordinates were as follows: Kitope (S. h. - 47.9\%, GPS - S 06 00.941' E 039 $\left.{ }^{\circ} 15.129^{\prime}\right)$, Mwera (S. h. - 29\%, GPS - S 06 08.629' E 039 ${ }^{\circ}$ 16.334'), Pwani Mchangani (S. h. - 1.8\%, GPS - S $05^{\circ}$ 55.228', E $\left.039^{\circ} 21.358^{\prime}\right)$, Fujoni (S. h. - 7.3\%, GPS - S $06^{\circ}$ 09.470', E $039^{\circ} 12.344^{\prime}$ ) and Mto Pepo (S. h. - 3.1\%, GPS $\left.06^{\circ} 08.770^{\prime}{\mathrm{E} 039^{\circ}}^{\circ} 13.292^{\prime}\right)$. The prevalence of $S$. haematobium reported here was assessed during May 2005, approximately one year after initial mass de-worming within schools took place. Mto Pepo school is within a semi-urban setting while all other schools were in rural environments. Kitope and Mwera schools fall well within the local endemic zone of S. haematobium.

Design of KA questionnaire - To assess children's KA for schistosomiasis a standardized written questionnaire was designed to be completed by each child under the supervision of the classroom teacher. The written questionnaire was translated into Kiswahili and pre-tested in two other primary schools (Chaani and Mahonda) to resolve ambiguities in translation and ascertain its performance against verbal interview technique (data not shown). The final questionnaire used a combination of both simple 'yes' or 'no' answers as well as multiple choice responses. A total of 18 questions were posed which were assigned to one of three separate sections: section $1-4$ questions (1.0. to 1.3.) on KA on general awareness of local diseases, section $2-7$ questions (2.0. to 2.6.) on KA for malaria and section $3-7$ questions (3.0. to 3.6.) on KA for urinary schistosomiasis. The questions were developed upon the messages to be found within Juma na Kichocho. The questionnaire forms can be downloaded in English or Kiswahili formats from: http://www. nhm.ac.uk/hosted_sites/schisto/jrs/unguja.html.

To assess immediate and putative booklet-induced changes in KA for schistosomiasis care was taken to design an equivalent set of questions for malaria. By using such a comparison a better assessment could be made for confounding changes resultant from re-interview (i.e. general improvement in questionnaire responses through familiarisation) or by increasing awareness from subsequent within-school discussions amongst school peers and teachers as well as within children's families. As an additional control for assessing putative booklet induced changes, one school acted as a local temporal control (see below).

Design of study - Within each school a total of 55 schoolchildren were selected including approximately equal number of boys and girls of between 11 and 15 years of age. Before booklets were given out to the class the KA questionnaire was implemented to record initial KA responses before booklet use. The booklets were then introduced to the children and a 30 min presentation, given by HCLU health educator, discussed their use set within a context of broader health issues concerning urinary schistosomiasis. Over a working school week, the classroom teacher then guided the children in their daily structured-use of the booklets, typically 10-15 min each day, and also encouraged the children to further inspect the booklets during school session breaks. At the end of a working school week, the same children were re-assessed by re-implementation of the same KA questionnaire as a two-time point comparison, i.e. before and after. As an additional control for assessing putative-booklet induced changes, the KA questionnaire was implemented 3 times at Kitope school; once at baseline, then again after a week period without use of booklets (to test for non-booklet induced changes) and a final third time after a one week using the booklets (to test for putative booklet induced changes).

Statistical analysis - Responses to questionnaires inputted into electronic format using EpiData v3 electronically exported to Stata v8 (StataCorp LP, Texas, US) for percentage tallies and for univariate statistical tests as well as numerical analyses such as Odds Ratios. To facilitate comparisons a variety of weighting schemes for the responses were investigated. A final scoring system was chosen such that a correct response was awarded a single positive mark while an incorrect response scored no mark; 
in multiple choice answers quarter, half and three quarters marks were allocated for partially correct answers. For each child a KA score was obtained for schistosomiasis and malaria by tallying correct responses to questions 3.0/2.0,3.1/2.1,3.2/2.2,3.3/2.3 and 3.6/2.6 such that each child's KA score could range from $0-5$. By progressively pooling children's KA scores by sex and then by schools a global total KA score could be obtained. Changes in the frequency distribution of global KA scores before and after booklet intervention were assessed by KolmogorovSmirnov tests. The two questions 2.4/3.4 and 2.5/3.5 concerning whether the child knew of anyone who had either disease or had previously taken treatment were not included in the total KA score as it was assumed that these responses would not be contingent upon use of booklets.

Ethical requirements - The study approved by the Ministry of Health and Social Welfare, Zanzibar and The London School of Hygiene and Tropical Medicine research ethics committee application number $04 / 314$.

\section{RESULTS}

At the end of the study period KA responses were available for analysis from a total of 229 children (114 boys : 115 girls) owing to drop-out of students and the rejection of partially completed questionnaires. The answers to section 1 provided useful background information to the responses to other sections within the local context of each school.

The reported occurrence of the major diseases across the five schools is shown in Fig. 1, with malaria being most common with approximately equal numbers of boys and girls while a strong sex bias was observed for kichocho $\left(\chi^{2}-14.1,1\right.$ d.f., $\left.p<0.001\right)$. Eye complaints, skin and stomach problems were commonly reported and assigned into the 'other' category. A total of $81.6 \%$ of the children reported that they had either been to a hospital or health centre and had received some form of treatment. About $90.9 \%$ of children were aware that health services teams had visited their school for which the most common re-

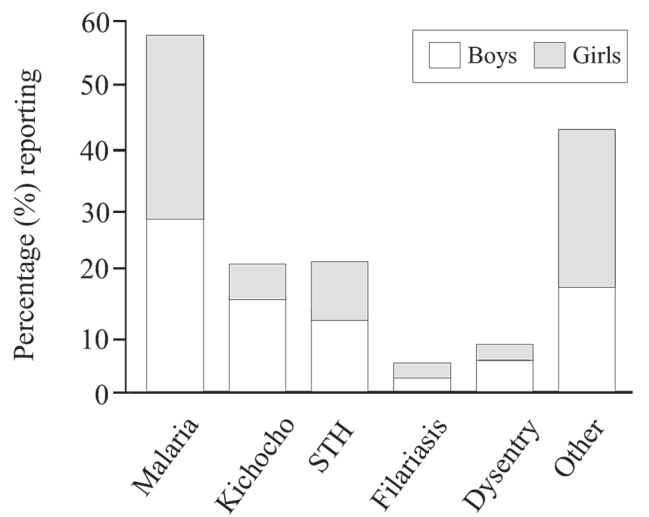

Fig. 1: the percentage of diseases reported by the students at questionnaire baseline. Malaria was most commonly reported and a strong sex bias was seen for students reporting kichocho. This likely reflects the local importance of malaria as well as known infection bias of boys, through increased water contact, for urinary schistosomiasis.

ported activities were in descending order: urine testing, administration of medications (tablets), stool testing, health questionnaires, administration of medications (injections) and health education talks. Across the five schools, kichocho was reported to be most common in Mwera (18.5\%), Kitope (15.6\%), Pwani Mchangani (14\%), Mto Pepo (10.5\%), and Fujoni (7.5\%).

With regard to KA questions for kichocho and malaria, the percentage of correct, not partially correct, answers before and after booklet intervention for kichocho and malaria is shown in the Table. A general positive increase was observed across all questions for the study period with exceptions of 2.1 and 2.6 which were concerned with malaria. In total $47.4 \%$ of children were already aware that schistosomiasis was a water-borne disease while only $10.5 \%$ knew of its exact aetiology; after booklet intervention these levels increased to 54.6 and $15.7 \%$, respectively. Responses to question 3.1 (signs and symptoms) and 3.3 (protection methods) showed great-

TABLE

Percentage of correct responses for knowledge and atitude questions before and after use of booklets

\begin{tabular}{|c|c|c|c|}
\hline Question & $\begin{array}{c}\text { Before booklet } \\
\% \text { correct }\end{array}$ & $\begin{array}{c}\text { After booklet } \\
\% \text { correct }\end{array}$ & Odds ratio increase $(95 \% \mathrm{CI})$ \\
\hline 3.0 What is kichocho $(\mathrm{K})$ ? & $10.5^{a}$ & 15.7 & $1.6(0.9-2.8)$ \\
\hline 2.0 What is malaria $(\mathrm{M}) ?^{a}$ & $7.1^{a}$ & 7.9 & $1.1(0.6-2.3)$ \\
\hline 3.1 What are the main signs and symptoms of $\mathrm{K}$ ? ${ }^{b}$ & 5.2 & 10.0 & $2.0(1.0-4.2)$ \\
\hline 2.1 What are the main signs and symptoms of $\mathrm{M}{ }^{b}$ & 6.1 & 5.7 & $0.9(0.4-2.0)$ \\
\hline 3.2 How is K caught? & 35.4 & 44.1 & $1.4(1.0-2.1)$ \\
\hline 2.2 How is M caught? & 73.4 & 84.4 & $1.8(1.1-2.7)$ \\
\hline 3.3 How can you protect yourself against $\mathrm{K}$ ? ${ }^{b}$ & 2.6 & 5.2 & $2.0(0.8-5.6)$ \\
\hline 2.3 How can you protect yourself against $\mathrm{M}^{b}$ & 3.5 & 4.8 & $1.4(0.6-3.6)$ \\
\hline 2.6 After treatment, can K ever be caught again? & 8.3 & 11.8 & $1.4(0.8-2.6)$ \\
\hline 3.6 After treatment, can $\mathrm{M}$ ever be caught again? & 19.7 & 16.2 & $0.8(0.5-1.2)$ \\
\hline
\end{tabular}

$a$ : note that $35 \%$ of children understood that kichocho was a disease transmitted by snails and that $48 \%$ of children understood that malaria was a disease transmitted by predominately night-biting mosquitoes but they were not fully aware of the diseases exact parasitic aetiologies; $b$ : these questions were multiple choice; only the correct and not partially correct percentage responses are shown. 
est increases. The percentage positive responses to the two questions 2.4/3.4 and 2.5/3.5 concerning whether the child knew of anyone who had either disease or had previously taken treatment were 62.4 and $47.1 \%$ and 57.8 and $57.2 \%$, respectively.

KA scores for schistosomiasis by school were plotted for before and after use of the booklets, Fig. 2. While the mean KA score for schistosomiasis increased from 1.52 (95\% CI $1.39-1.64)$ to $1.73(95 \%$ CI $1.60-1.86)$ that for malaria also followed a positive trend 1.97 (95\% CI $1.84-$ $2.09)$ to $2.13(95 \%$ CI $2.01-2.25)$ and a Mann-Whitney UTest showed that these slight increases were non-significant for all schools. During the temporal control for Kitope school, mean KA scores at baseline, 1-week later without use of booklets and a further 1-week later with use of booklets were 1.81 (95\% CI 1.53-2.08), 1.95 (95\% CI 1.67 $-2.22)$, and 2.09 ( $95 \%$ CI $1.75-2.43)$, respectively.

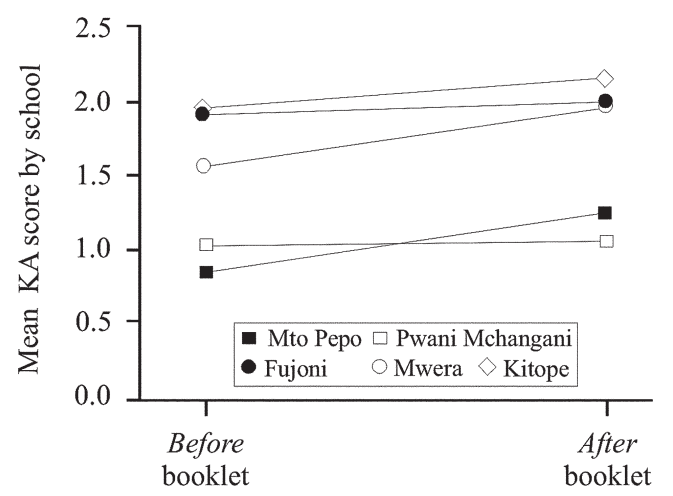

Fig. 2: the dynamics of mean knowledge and attitude scores for kichocho by school for before and after booklet intervention; although positive gains could be seen for each school these were nonsignificant. (Apart from Mto Pepo, all schools are found within rural environments with both Mwera and Kitope located well within the endemic zone for urinary schistosomiasis.)

The frequency distributions of global KA scores for schistosomiasis and malaria before and after the intervention is shown in Fig. 3. While positive increases in KA scores could be seen for both diseases, KolmogorovSmirnov comparisons revealed non-significant changes in KA for either disease over the study period.

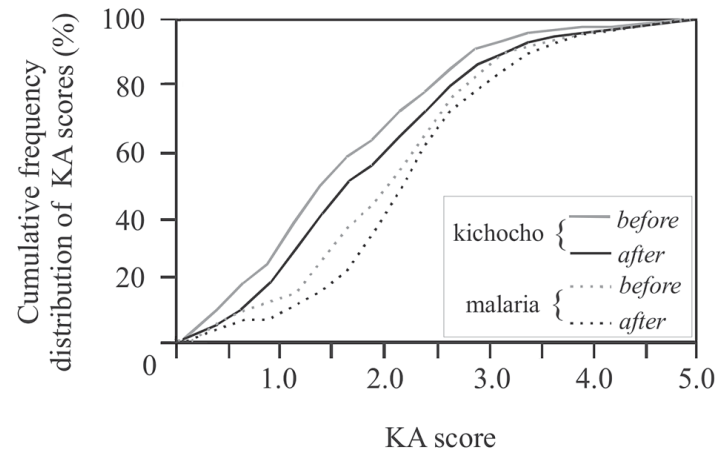

Fig. 3: cumulative frequency distribution plot of total knowledge and attitude scores for kichocho and malaria before and after booklet intervention. While increases were observed (evidenced by rightshift of curves) for both diseases these changes were not statistically significant by Kolmogorov-Smirnov tests.

\section{DISCUSSION}

Health education is an essential facet within effective strategies for integrated control of parasites, especially in the context of helminth control (Asaolu \& Ofoezie 2003). On Unguja there have been extensive efforts placed on the control of malaria focusing upon the more widespread use of better first-line anti-malarials as well as distribution and implementation of impregnated mosquito bednets (Curtis \& Mnzava 2000, Simooya 2005). Concurrent with these activities has been a programme of raising public awareness across the island (Alilio et al. 1998) and numerous health education posters and placards are continuously on display e.g. within health centres and upon road side locations. By taking advantage of this public outreach, these efforts could be useful as a comparative 'yardstick' in assessing the ongoing health education impact upon the Ungujan populace by other control programmes.

For kichocho, public awareness of the disease has been shown to be low and in need of improvement by health education and political advocacy (Stothard et al. 2002b). With the presidential launch and the opening ceremony of the Kick out Kichocho programme, which featured on local television, significant steps have been taken to promote political advocacy and general awareness of the programme at all levels. For the schoolaged child, however, health education is still required to enforce these messages to make children better aware of the dangers of kichocho and to initiate behavioural change. Measuring the tangible impact of health education can be problematic, especially if the timeframe for monitoring is limited and that there is no simple relation between health education and reduction of parasitaemia (Schall 1989, 1995, Kloos 1995, Schall \& Diniz 2001, Lansdown et al. 2002). Nevertheless, it is still important to make formal assessment of the impact of health education especially, in terms of costeffectiveness, when prioritisation of funds available for control is required. This is particularly true when the health education costings are to be taken from vertical programmes whose budgets are primarily focused upon provision and administration of anthelmintics.

From first appearances the Juma na Kichocho is a child friendly booklet which without doubt captures the immediate interest of the children upon initial exposures to it. The booklet is carefully loaded with key images and messages which are tailor-made for IEC purposes. We have observed that the children's reactions to the booklets were strongly positive. Over $80 \%$ of the children found the booklet to be interesting, fun to use and felt that they learnt something new from it (data not shown) which would confirm an appropriate cultural delivery (see Kloos 1995). The more formal evaluation using the KA questionnaires revealed, however, a rather disappointing tangible impact even in the light of the short time frame but high booklet exposure of this pilot study. From the two time points of before and after, as well as the temporal control school at Kitope, it was shown that the general increase in KA scores was not statistically significant. In addition the similar positive trajectory for KA scores for malaria strongly suggest that any increase will be partially resultant from the re-administration of the question- 
naire such that, after baseline, the children probably discussed issues pertinent to the questionnaire amongst their school peers and teachers as well as at home with family and friends and in so doing likely raised their KA scores. While the mean Odds Ratio increase across the 5 KA questionnaire for schistosomiasis was 1.68 that for malaria was 1.20 so gains associated with the Juma na Kichocho booklet, while detectable, cannot be considered to be strong.

Judged against a general yardstick of KA for malaria, existing KA scores were lower for kichocho which would be expected given the current public outreach of the malaria control programme (Alilio et al. 1998) and that the acute severity of the disease captures greater attention. In addition, children reported more frequently that they knew someone who had had malaria (62.4\%) than kichocho $(47.1 \%)$ which would also likely raise immediate awareness and understanding. Of particular note, however, is that $13.5 \%$ of the children in the survey still had no conception that malaria was a mosquito-borne disease while $16.6 \%$ had no conception that kichocho was a water-borne disease. It would appear that there will remain a minority who continue to be non-receptive to health education messages, in common with observations of other programmes (Sow et al. 2003, Poggensee et al. 2005). This is particularly important when set against the aims of disease transmission reduction as the potential gains made with altered behaviours by those receptive to health education messages, however small, might be offset against those who were not (Teesdale 1986). In this light it is relevant to highlight that the majority of children still failed to realise the re-infection risk after treatment.

Around 10,000 booklets have been allocated to Zanzibar equally assigned to Unguja and Pemba Islands. So far on Unguja 55 booklets have been distributed to each of 70 primary schools where ongoing de-worming activities are taking place. Within a typical Ungujan primary school there are between 800-1500 pupils so provision of 55 booklets per school is usually sufficient to provide a standard class with access to the booklets if pupils work in student pairs. The high quality of the booklet is good for durability and long-term use but this is also reflected in the price. When taking production, shipping and delivery into account, the cost may be over 5 USD per booklet; a substantial cost compared to treatment with praziquantel, which is now less than 0.1 USD (Molyneux et al. 2005).

Integration of this booklet into the classroom curriculum and rotating the use of booklets between classes will ensure that all children will have exposure to the IEC messages. To reinforce these messages, health education talks, supplemental to the booklets will hopefully improve impact. In close liaison with teachers in Unguja a programme of booklet implementation and structured-use is to be developed to maximise the educational impact. This pilot study suggests that the booklet does not provide a significant change in knowledge and attitude in the short term and reinforces the idea that long term education is required to promote and guide behavioural change, especially in relation to reduction of environmental water contact and good health practises. Only by minimizing water contact and improving hygiene will re-infection be reduced or avoided, thus prolonging the beneficial impacts of treatment with praziquantel.

\section{ACKNOWLEDGEMENTS}

To the technical support and field staff of the Helminth Control Laboratory Unguja for their assistance during this study as well as to the teachers and children who took part. The Kick out Kichocho programme receives support from the Healt Foundation, the African Development Bank and Schistosomiasis Control Initiative.

\section{REFERENCES}

Albonico M, Chwaya HM, Montresor A, Stolfzfus RJ, Tielsch JM, Alawi KS, Savioli L 1997. Parasitic infections in Pemba Island schoolchildren. East Afr Med J 74: 294-298.

Alilio MS, Eversole H, Bammek J 1998. A KAP study on malaria in Zanzibar: implications for prevention and control A study conducted for UNICEF Sub-Office Zanzibar. Eval Program Plann 21: 409-413.

Aryeetey ME, Aholu C, Wagatsuma Y, Bentil G, Nkrumah FK, Kojima S 1999. Health education and community participation in the control of urinary schistosomiasis in Ghana. East Afr Med J 76: 324-329.

Asaolu SO, Ofoezie IE 2003. The role of health education and sanitation in the control of helminth infections. Acta Trop 86: 283-294.

Curtis CF, Mnzava AEP 2000. Comparison of house spraying and insecticide-treated nets for malaria control. Bull WHO 78: 1389-1400.

Engels D, Mpitabakana P 1989. Schistosomiasis control and health-education in Burundi. Trop Med Parasitol 40: 226227.

Fenwick A 2006. New initiatives against Africa's worms. Trans $R$ Soc Trop Med Hyg 100: 200-207.

King CH, Dickman K, Tisch DJ 2005. Reassessment of the cost of chronic helmintic infection: a meta-analysis of disability-related outcomes in endemic schistosomiasis. Lancet 365: 1561-1569.

Kloos H 1995. Human-behavior, health-education and schistosomiasis control - A review. Soc Sci Med 40: 1497-1511.

Lansdown R, Ledward A, Hall A, Issae W, Yona E, Matulu J, Mweta M, Kihamia C, Nyandindi U, Bundy D 2002. Schistosomiasis, helminth infection and health education in Tanzania: achieving behaviour change in primary schools. Health Educ Res 17: 425-433.

Lwambo NJS, Savioli L, Kisumku UM, Alawi KS, Bundy DAP 1997. The relationship between prevalence of Schistosoma haematobium infection and different morbidity indicators during the course of a control programme on Pemba island. Trans R Soc Trop Med Hyg 91: 643-646.

Molyneux DH, Hotez PJ, Fenwick A 2005. "Rapid-impact interventions": how a policy of integrated control for Africa's neglected tropical diseases could benefit the poor. PLos Med 2: 1064-1070.

Poggensee G, Krantz I, Nordin P, Mtweve S, Ahlberg B, Mosha G, Freudenthal S 2005. A six-year follow-up of schoolchildren for urinary and intestinal schistosomiasis and soiltransmitted helminthiasis in Northern Tanzania. Acta Trop 93: 131-140.

Schall V, Diniz MCP 2001. Information and education in schistosomiasis control: an analysis of the situation in the state of Minas Gerais, Brazil. Mem Inst Oswaldo Cruz 96: 3543. 
Schall VT 1989. Health-education and schistosomiasis - A brief retrospective and a proposal. Mem Inst Oswaldo Cruz 84: 84-90.

Schall VT 1995. Health education, public information and communication in schistosomiasis control in Brazil - A brief retrospective and perspectives. Mem Inst Oswaldo Cruz 90: 229-234.

Simooya O 2005. The WHO 'roll back malaria project' - Planning for adverse event monitoring in Africa. Drug Saf 28: 277-286.

Southgate VR, Rollinson D, Tchuente LAT, Hagan P 2005. Towards control of schistosomiasis in sub-Saharan Africa. $J$ Helminthol 79: 181-185.

Sow S, de Vlas SJ, Mbaye A, Polman K, Gryseels B 2003. Low awareness of intestinal schistosomiasis in northern Senegal after 7 years of health education as part of intense control and research activities. Trop Med Int Health 8: 744-749.

Stothard JR, Rollinson D 1997. Molecular characterization of Bulinus globosus and B. nasutus on Zanzibar, and an investigation of their roles in the epidemiology of Schistosoma haematobium. Trans R Soc Trop Med Hyg 91: 353357.
Stothard JR, Mgeni AF, Khamis S, Seto E, Ramsan M, Hubbard SJ, Kristensen TK 2002a. Royal Society of Tropical Medicine and Hygiene Meeting at Manson House, London, 21 February 2002 - Fresh from the field - New insights into the transmission biology of urinary schistosomiasis in Zanzibar. Trans R Soc Trop Med Hyg 96: 470-475.

Stothard JR, Mgeni AF, Khamis S, Seto E, Ramsan M, Rollinson D 2002b. Urinary schistosomiasis in schoolchildren on Zanzibar Island (Unguja), Tanzania: a parasitological survey supplemented with questionnaires. Trans $R$ Soc Trop Med Hyg 96: 507-514.

Teesdale CH 1986. The role of health-education to reduce transmission of schistosomiasis. Trop Med Parasitol 37: 184185.

van der Werf MJ, de Vlas SJ, Brooker S, Looman CWN, Nagelkerke NJD, Habbema JDF, Engels D 2003. Quantification of clinical morbidity associated with schistosome infection in sub-Saharan Africa. Acta Trop 86: 125-139.

Yuan LP, Manderson L, Tempongko MSB, Wei WY, Aiguo P 2000. The impact of educational videotapes on water contact behaviour of primary school students in the Dongting Lakes region, China. Trop Med Int Health 5: 538-544. 